\title{
Mogamulizumab for the treatment of adult T-cell leukemia/lymphoma
}

This article was published in the following Dove Press journal:

Blood and Lymphatic Cancer: Targets and Therapy

18 December 2014

Number of times this article has been viewed

\section{Makoto Yoshimitsu Naomichi Arima \\ Division of Hematology and Immunology, Center for Chronic Viral Diseases, Graduate School of Medical and Dental Sciences, Kagoshima University, Kagoshima, Japan}

\begin{abstract}
Adult T-cell leukemia/lymphoma (ATLL) is a peripheral T-cell lymphoma caused by latent infection of human T-cell lymphotropic virus type 1 . The outcome for ATLL is very poor, with a 3 -year overall survival of approximately $24 \%$ with conventional chemotherapy; thus, there is an unmet need for developing new treatment options. Defucosylated humanized anti-CC chemokine receptor 4 (CCR4) antibody (KW-0761, mogamulizumab) has been clinically available for the treatment of relapsed or refractory ATLL in Japan since 2012, and a Phase II study of mogamulizumab for patients with relapsed CCR4+ATLL demonstrated a 50\% objective response, a 30.8\% complete response, and an acceptable safety profile. Allogeneic hematopoietic stem cell transplantation has been used to treat patients with ATLL, and mogamulizumab in combination with allogeneic hematopoietic stem cell transplantation has been used successfully in a limited number of patients to treat refractory or relapsed ATLL. The efficacy of combining mogamulizumab with standard chemotherapy (mLSG15) for patients with ATLL has also been examined, and the results have shown higher rates of complete response with the combined therapy (52\%) compared with for chemotherapy alone (33\%). Mogamulizumab also has potential application in the treatment of human T-cell lymphotropic virus type 1-associated myelopathy/tropical paraparesis, Epstein-Barr virus-associated T-cell and natural killer-cell lymphoproliferative diseases, and peripheral and cutaneous T-cell lymphomas. Possible adverse events of mogamulizumab have been reported, such as cutaneous adverse reactions (including Stevens-Johnson syndrome), diffuse panbronchiolitis, reactivation of hepatitis B, and opportunistic infections. The treatment outcome of patients with ATLL might be improved by further optimizing the use of mogamulizumab and managing severe adverse events. In this review, we provide an overview of the current treatment options for patients with ATLL and a focused review on the use, application, and adverse events associated with mogamulizumab.
\end{abstract}

Keywords: ATLL, anti-CCR4 antibody, defucosylated antibody, cutaneous adverse reaction

\section{Introduction}

Adult T-cell leukemia/lymphoma (ATLL), a highly aggressive peripheral T-cell lymphoma (PTCL) caused by human T-cell lymphotropic virus type-1, has a very poor prognosis. ${ }^{1-4}$ In a retrospective analysis of 807 patients newly diagnosed with acuteand lymphoma-type ATLL between January 2000 and May 2009, the median overall survival (OS) time was 7.7 months. ${ }^{5}$ Despite the fact that allogeneic hematopoietic stem cell transplantation (allo-HSCT) has recently been used to treat this disease and has demonstrated promising long-term survival, Medically well-fit patients and those with good disease control are able to benefit from this treatment modality. ${ }^{6}$ Population-based analyses predict that patients with ATLL in their 60s and 70s will make up two-thirds of all patients with ATLL over the next 15 years in Japan. Partly 
because of this aging patient population, it is obvious that new and less toxic treatments need to be developed. ${ }^{7}$ In this review, we provide an overview of the current treatment options for patients with ATLL, as well as a focused review on the use and application of and adverse events (AEs) associated with mogamulizumab.

\section{Current treatment options and prognosis}

Current treatment guidelines according to ATLL subtypes were discussed in an international consensus meeting, proposed previously, and are summarized in Table $1 .^{8}$

\section{Conventional cytotoxic chemotherapy}

Patients with ATLL are often administered conventional chemotherapy as frontline therapy. The results of a Phase III randomized control trial suggest that a regimen of vincristine, cyclophosphamide, doxorubicin, and prednisone (VCAP); doxorubicin, ranimustine, and prednisone (AMP); and vindesine, etoposide, carboplatin, and prednisone (VECP) is superior to biweekly cyclophosphamide, doxorubicin, vincristine, and prednisone (CHOP) in newly diagnosed aggressive (acute, lymphoma, or unfavorable chronic) types of ATLL, considering the complete response rate $(40 \%$ versus $25 \% ; P=0.020) .{ }^{9} \mathrm{OS}$ at 3 years was $24 \%$ in the VCAP-AMP-VECP group and $13 \%$ in the CHOP group $(P=0.085)$. Thus, the results of this randomized study indicate that the VCAP-AMP-VECP regimen should be recommended for further investigation. Nonetheless, the median survival time of 13 months remains unfavorable compared with the survival times of other hematologic malignancies.

Table I Recommended treatment strategy for ATLL subtypes before the mogamulizumab era

\begin{tabular}{|c|c|}
\hline ATLL subtype & $\begin{array}{l}\text { Recommended } \\
\text { treatment strategy } \\
\text { (outside clinical trials) }\end{array}$ \\
\hline $\begin{array}{l}\text { Smoldering-type (asymptomatic), } \\
\text { favorable chronic-type (asymptomatic) }\end{array}$ & Watch and wait \\
\hline $\begin{array}{l}\text { Smoldering-type (symptomatic), } \\
\text { favorable chronic-type (symptomatic) }\end{array}$ & AZT/IFN or watch and wait \\
\hline Unfavorable chronic-type, acute-type & $\begin{array}{l}\text { Chemotherapy or AZT/IFN } \\
\text { Allogeneic hematopoietic } \\
\text { stem cell transplantation }\end{array}$ \\
\hline Lymphoma-type & $\begin{array}{l}\text { Chemotherapy } \\
\text { Allogeneic hematopoietic } \\
\text { stem cell transplantation }\end{array}$ \\
\hline
\end{tabular}

Abbreviations: ATLL, adult T-cell leukemia/lymphoma; AZT, zidovudine; IFN, interferon.

\section{Allo-HSCT}

Allo-HSCT has been explored as a promising alternative therapeutic modality that can provide long-term response in a proportion of patients with ATLL. ${ }^{10-12}$ In a recent large nationwide retrospective analysis, investigators compared the outcomes of 386 patients with ATLL who underwent allo-HSCT and found that after a median follow-up of 41 months, the 3-year OS for the entire cohort was 33\%. ${ }^{12}$ Another retrospective analysis of 294 patients with ATLL who received allo-HSCT revealed that the development of grade I-II acute graft-versus-host disease (GVHD) confers a lower risk of disease progression and a better OS, which is indicative of the presence of a graft-versus-leukemia effect. ${ }^{13}$ Another large retrospective analysis of allo-HSCT for ATLL $(n=586)$ in Japan observed no significant difference in OS by intensity of conditioning regimen; however, there was a trend suggesting that reduced-intensity conditioning regimens contributed to better OS in older patients. ${ }^{14}$ The number of patients with ATLL eligible for allo-HSCT is quite limited because of the older age at diagnosis and the lower rate of induction of a complete response (CR). Selection criteria for allo-HSCT for patients with ATLL remain undetermined.

\section{Interferon- $\alpha$ and zidovudine}

In recent years, the combined use of interferon $\alpha$ (IFN- $\alpha$ ) and zidovudine (AZT) to treat ATLL has been proposed. The results of a recent meta-analysis on the use of AZT/ IFN for 254 patients with ATLL showed that this treatment resulted in a better response and a prolonged OS. ${ }^{15}$ In this study, 207 patients received first-line chemotherapy, antiviral therapy, or chemotherapy followed by antiviral therapy. For the 75 patients in this study who received first-line antiviral therapy, the 5-year OS rate was $46 \%(P=0.004)$. Furthermore, in acute ATLL, achievement of CR with antiviral therapy resulted in an $82 \% 5$-year survival. These results suggest that treatment of ATLL using AZT/IFN results in high response and $\mathrm{CR}$ rates, resulting in prolonged survival in a significant proportion of patients; the exception is for cases of lymphoma-type ATLL. Although this was a retrospective analysis, the results seem to be promising, and further studies comparing AZT/IFN- $\alpha$ and conventional chemotherapy or allo-HSCT are warranted.

\section{Monoclonal antibodies}

The use of monoclonal antibodies for the treatment of hematological malignancies has evolved into a promising approach since the FDA approved the anti-CD20 antibody, rituximab, for malignant lymphoma in 1997. Rituximab markedly improved 
the prognosis of patients with CD20+ B-cell lymphoma and changed the standard therapy for this malignancy. ${ }^{16}$ This success of monoclonal antibody therapy in the field of lymphoma is prompting researchers to search for new effective monoclonal antibody therapies for T-cell lymphoma. Cell surface antigens, tumor-specific antigens, and antigens with less off-target effects are assumed to be ideal target antigens for antibody therapy.

\section{The CC chemokine receptor 4 and defucosylated antibody}

One target of molecular therapy is chemokine receptors. Chemokines are a family of small, structurally related cytokines. The chemokines play a crucial role in cell migration and homing of various lymphocyte subpopulations. All known chemokine receptors are seven-transmembrane $G$ protein-coupled receptors. CC chemokine receptor 4 (CCR4) is the receptor for CCL17 and CCL22. CCR4 is known to be expressed on $\mathrm{Th}_{2}$ cells, cutaneous lymphocyte antigenpositive skin-homing cells, $\mathrm{T}_{\text {reg }}$ cells, $\mathrm{Th}_{17}$ cells, $\mathrm{Th}_{22}$ cells, $\mathrm{Tc}_{2}$ cells, interleukin 2-activated natural killer (NK) cells, a small subset of primary NK cells, CD4+ NK cells, airway eosinophils, and platelets. ${ }^{17}$

As various T-cell subsets differentially express chemokine receptors, Yoshie et al used the chemokine receptor expression pattern to determine the cellular origin and T-cell neoplasm-specific antigens. ${ }^{18}$ They found a very high expression of CCR4 on ATLL cells and human T-cell lymphotropic virus type-1-immortalized T cells. ${ }^{18}$ Ishida et al also performed an extensive study of CCR4 on formalin-fixed and paraffin-embedded samples from 103 patients with ATLL and reported that tumor cells from $88.3 \%$ of these patients expressed CCR $4 .{ }^{19}$ On the basis of these findings, CCR4 was selected as a novel molecular target for monoclonal antibody therapy in ATLL.

Monocytes and NK cells are the main effector cells of antibody-dependent cellular cytotoxicity (ADCC), which requires the presence of activating $\mathrm{Fc}$ receptors on the effector cells. ADCC is believed to be an important therapeutic mechanism of clinically effective antibodies ${ }^{20}$ The presence and profiles of oligosaccharides in the Fc region are known to play an important role in ADCC. Shields et al reported that the binding of fucose-deficient immunoglobulin G1 to human FcgammaRIIIA improved up to 50-fold. ${ }^{21}$ Assays measuring ADCC activity using purified peripheral blood monocytes or NK cells from several donors showed enhanced cytotoxic function, even with a very low antibody concentration. ${ }^{21}$ Similarly, Niwa et al reported that defucosylated anti-CCR4 immunoglobulin G1 antibody reduced the antigen amount on target cells required for the same level of ADCC induction by 10 -fold. ${ }^{22}$

\section{KM2760 and mogamulizumab (KW-076 I): preclinical testing}

With this ADCC-enhancing defucosylation technology, the therapeutic anti-CCR4 monoclonal antibody (KM2760) was developed for the treatment of ATLL. ${ }^{23}$ KM2760 was shown to exhibit much higher ADCC activity, using human peripheral blood mononuclear cells (PBMCs) as effector cells against ATLL cells, compared with the highly fucosylated anti-CCR4 antibody. In addition, KM2760 exhibited potent ADCC in the presence of lower concentrations of human PBMCs. Ishida et al further demonstrated that KM2760 induced ADCC against primary ATLL cells in an autologous setting, suggesting that the ATLL patients' PBMCs retain substantial ADCC-effector function. ${ }^{24}$ These promising preclinical data led to the development of this antibody for clinical application.

Defucosylated humanized anti-CCR4 antibody (mogamulizumab, KW-0761) was then developed by replacing mouse immunoglobulin constant regions and variable domains with the human equivalents; ${ }^{25}$ replacing the constant regions affects the largest reduction in immunogenicity, and humanization of variable domains affects a further decrease. ${ }^{25} \mathrm{KW}-0761$ showed potent antitumor activity mediated by highly enhanced ADCC against primary ATLL cells in an autologous setting. The degree of KW-0761 ADCC was mainly determined by the amount of effector NK cells present, but not the amount of the target molecule CCR4 on the ATLL cell surface. The anti-ATLL effect of this antibody was further evaluated in mice models of ATLL. ${ }^{24-27}$ Among ATLL mouse models, Ito et al established a primary mouse model using ATLL-cell-bearing NOD/Shi-scid, interleukin 2R gamma null mice in which autologous human immune cells were engrafted. ${ }^{27}$ In this excellent model, the anti-ATLL effect of the therapeutic antibody against primary ATLL cells was proved to be mediated by autologous human immune cells.

\section{Clinical trials and case reports of patients with adult T-cell leukemia/lymphoma treated with mogamulizumab} Phase I study

On the basis of the above promising preclinical studies, a clinical Phase I study of mogamulizumab was conducted. 
The Phase I study assessed the safety, pharmacokinetics, recommended Phase II dose, and efficacy of mogamulizumab in patients with relapsed CCR4+ ATLL or PTCL. ${ }^{28}$ Sixteen patients received mogamulizumab once a week for 4 weeks intravenously. Doses were escalated, going from 0.01 , to 0.1 , to 0.5 , and to a final concentration of $1.0 \mathrm{mg} / \mathrm{kg}$. Fifteen patients completed the protocol treatment. The maximum tolerated dose was not reached. Therefore, the recommended Phase II dose was determined to be $1.0 \mathrm{mg} / \mathrm{kg}$. No patients had detectable levels of antimogamulizumab antibody. Five patients achieved objective responses: two CRs and three partial responses.

\section{Phase II study}

A multicenter Phase II study of mogamulizumab for patients with relapsed, aggressive CCR4+ ATLL was conducted to evaluate the efficacy, pharmacokinetic profile, and safety of this agent. ${ }^{29}$ The primary end-point was the overall response rate, and the secondary end-points included progression-free survival and OS from the first treatment. Patients received mogamulizumab once per week for 8 weeks at a dose of $1.0 \mathrm{mg} / \mathrm{kg}$. Objective responses were observed in 13 of 26 patients, including eight CRs, with an overall response rate of $50 \%$ (95\% confidence interval, 30\%-70\%). The median progression-free survival and OS were 5.2 and 13.7 months, respectively. The mean half-life period after the eighth infusion was $422 \pm 147$ hours ( \pm standard deviation).

\section{Allogeneic hematopoietic stem cell transplantation with mogamulizumab}

To date, there have been only three case reports of patients with ATLL who received allo-HSCT and mogamulizumab. ${ }^{30-32}$ Mogamulizumab was administered before alloHSCT in two of the cases. ${ }^{30,31}$ Both cases were refractory to conventional chemotherapy, and initiation of mogamulizumab induced CR at the time of allo-HSCT. As the achievement of CR at allo-HSCT is one of the most important prognostic factors, ${ }^{14}$ administration of mogamulizumab to patients with chemotherapy-resistant ATLL might improve their disease status before allo-HSCT and result in improved survival. There is just a single reported case of successful treatment with mogamulizumab for relapsed ATLL after umbilical cord blood transplantation. ${ }^{32}$ Mogamulizumab decreases CCR4-expressing regulatory T cells (Tregs), and it is postulated that a decreased level of Tregs before and after allo-HSCT might increase the incidence of GVHD. In one report by Ohgiya et al, elevation of the total bilirubin level and the development of massive ascites on day 40 were observed, and the patient died of organ failure on day $110 .{ }^{30}$ Even though the authors of that case considered the cause of death regimen-related toxicity, and despite the fact that all three reported cases of allo-HSCT with mogamulizumab did not show grade III-IV GVHD, the safety profile of mogamulizumab before and after allo-HSCT, including the severity of GVHD, should be examined in a larger series of patients. The role of mogamulizumab in patients who could be eligible for allogeneic transplant also requires further evaluation.

\section{Combination therapy of mogamulizumab with chemotherapy}

A multicenter, randomized, Phase II trial was conducted to examine the efficacy of the combination of mogamulizumab with standard chemotherapy (mLSG15) for ATLL. Patients with previously untreated aggressive ATLL received four courses of an mLSG15 regimen, with or without mogamulizumab $(1.0 \mathrm{mg} / \mathrm{kg})$, once every 2 weeks, to achieve a total of eight doses. The rate of CR in mLSG15 with and without mogamulizumab was $52 \%$ and $33 \%$, respectively. ${ }^{33}$ In the near future, this combination therapy of mogamulizumab with mLSG15 may be a potent standard treatment option for patients with newly diagnosed aggressive ATLL.

These results of clinical trials are summarized in Table 2.

Table 2 Summary of clinical trials of mogamulizumab

\begin{tabular}{|c|c|c|c|c|}
\hline Clinical trial & Subject & Mogamulizumab & Results & Reference \\
\hline $\begin{array}{l}\text { Phase I study } \\
(\mathrm{n}=16)\end{array}$ & Relapsed CCR4+ ATLL or PTCL & $0.01-1.0 \mathrm{mg} / \mathrm{kg}$ weekly & $\begin{array}{l}\text { Five patients ( } 3 \mid \%) \text { had an objective } \\
\text { response (two CR, three PR) }\end{array}$ & 28 \\
\hline $\begin{array}{l}\text { Phase Il study } \\
(n=26)\end{array}$ & Relapsed CCR4+ ATLL & $1.0 \mathrm{mg} / \mathrm{kg}$ weekly & $\begin{array}{l}13 \text { patients }(50 \%) \text { had an objective } \\
\text { response, including eight CR }\end{array}$ & 29 \\
\hline $\begin{array}{l}\text { Phase II study } \\
(\mathrm{n}=53)\end{array}$ & Untreated CCR4+ ATLL & $\begin{array}{l}\text { Chemotherapy with (group A) or } \\
\text { without (group B) mogamulizumab }\end{array}$ & $\begin{array}{l}29 \text { patients }(52 \%) \text { in group } A \text { and } \\
24 \text { patients }(33 \%) \text { in group B had a CR }\end{array}$ & 33 \\
\hline
\end{tabular}

Abbreviations: CCR4, CC chemokine receptor 4; ATLL, adult T-cell leukemia/lymphoma; PTCL, peripheral T-cell lymphoma; CR, complete response; PR, partial response. 


\section{Other applications \\ of mogamulizumab \\ Preclinical: Epstein-Barr \\ virus-associated T-cell and NK-cell \\ lymphoproliferative diseases}

T-cell and NK-cell lymphoproliferative diseases can be refractory to conventional chemotherapy. The Epstein-Barr virus (EBV) infects not only B cells but also $T$ cells and NK cells. Kanazawa et al demonstrated CCR4 expression in most EBV-positive $\mathrm{T}$ and NK cell lines, and mogamulizumab induced ADCC activity against CCR4+ cell lines, inhibiting the growth of EBV-positive NK-cell lymphomas in a murine xenograft model. ${ }^{34}$ Furthermore, CCR4 was expressed on EBV-infected cells in eight of 17 patients with EBV-associated T-cell and NK-cell lymphoproliferative diseases. These results indicate that mogamulizumab may be a therapeutic option for EBV-associated T-cell and NK-cell lymphoproliferative diseases.

\section{Peripheral T-cell lymphoma and cutaneous T-cell lymphoma}

The Phase I study of mogamulizumab for patients with ATLL also included three relapsed patients with PTCL. ${ }^{28}$ In addition, a multicenter Phase II study of mogamulizumab for patients with relapsed PTCL and cutaneous T-cell lymphoma was conducted in Japan. ${ }^{35}$ Mogamulizumab $(1.0 \mathrm{mg} / \mathrm{kg}$ ) was administered intravenously once a week for 8 weeks. An objective response was observed in 13 of 37 patients (35\%; 95\% confidence interval, 20\%-53\%), including $14 \%$ with CR. The most common AEs were hematologic events, pyrexia, and cutaneous AEs, all of which were manageable.

\section{AEs associated with mogamulizumab Phase I and Phase II study AEs}

In the Phase I study of mogamulizumab for patients with relapsed ATLL and PTCL, only one of 15 patients developed grade 3 dose-limiting toxicities of skin rash and febrile neutropenia and grade 4 neutropenia. ${ }^{28}$ Other treatmentrelated grade 3-4 toxicities included lymphopenia $(n=10)$, neutropenia $(n=3)$, leukopenia $(n=2)$, herpes zoster infection $(\mathrm{n}=1)$, and acute infusion reaction/cytokine release syndrome $(\mathrm{n}=1)$. In the Phase II study, the most common nonhematologic $\mathrm{AE}$ was an infusion reaction (89\%). ${ }^{28}$ The infusion reaction occurred primarily at the first infusion, becoming less frequent with subsequent treatments. Skin rashes were observed as another frequent nonhematologic AE (63\%), mostly occurring after the fourth or subsequent infusions. Of the 14 patients who developed grade 2 or higher skin rashes, objective responses were noted in 13 patients (93\%), including eight CRs. In contrast, of the twelve patients who developed no or grade 1 skin rashes, no objective responses were observed. Lymphopenia occurred in 26 (96\%) of the 27 patients. Grades 3-4 thrombocytopenia was observed in five patients (19\%) but was not associated with bleeding, and grade 3 neutropenia also occurred in five patients but did not lead to a febrile episode.

\section{Cutaneous adverse reactions}

The interim report for the postmarketing surveillance from May 29 to September 28, 2012, revealed nine skinrelated severe AEs, including four cases of Steven-Johnson syndrome/toxic epidermal necrolysis. ${ }^{36}$ Among four cases with Steven-Johnson syndrome, there was a durable significant reduction of the CD4+CD25highFOXP3+ Treg cell subset in the patient's PBMC, and the affected inflamed skin almost completely lacked FOXP3+ cells. This implies an association between reduction of the Treg subset by mogamulizumab and occurrence of Steven-Johnson syndrome. Yonekura et al also reported two cases of ATLL with cutaneous adverse reactions. In the skin lesions of the two patients, CD4+CD8-CD25+ ATLL cells were replaced by CD3+8+ cytotoxic $\mathrm{T}$ cells. Cutaneous adverse reactions developed in two patients with CR, who did not show a relapse of ATLL over the course of 9 months; thus, cutaneous adverse reactions are suggested to be markers for a favorable prognosis. ${ }^{37}$

\section{Reactivation of hepatitis B}

Nakano et al reported hepatitis B virus (HBV) reactivation in a patient with ATLL with a previously resolved HBV infection after mogamulizumab treatment. ${ }^{38}$ Even though the direct association of mogamulizumab treatment and HBV reactivation is not adequately clear, further attention needs to be focused on this potentially fatal AE.

\section{Opportunistic infection}

Ohyama et al reported two cases of cytomegalovirus infection (fatal encephalitis and colitis) in four elderly patients after mogamulizumab treatment. ${ }^{39}$ In Phase I and Phase II trials, lymphopenia was a major AE..$^{27,28}$ Therefore, prophylactic treatment for opportunistic infection, including cytomegalovirus infection, needs to be carefully assessed in further studies. 


\section{Diffuse panbronchiolitis}

There has been a single reported case of a patient with relapsed ATLL complicated with diffuse panbronchiolitis 6 months after mogamulizumab treatment. ${ }^{40}$ In this case, the number of CD4+CD25+FOXP3+ cells was low, and CD8+ cells had increased just before the onset of diffuse panbronchiolitis. CD8+ cells and neutrophils, together with inflammatory cytokines, are believed to play key roles in the development of diffuse panbronchiolitis. Pulmonary involvement might develop because of mogamulizumab as a result of an immune response of $\mathrm{CD} 8+$ cells that are not suppressed by Tregs. Because late-onset AEs related to mogamulizumab treatment remain unclear as a result of the short observation period and the small number of treated patients, physicians should be aware of possible rare complications.

\section{Conclusion}

Treatment options for patients with ATLL are expanding beyond conventional cytotoxic chemotherapy to include allo-HSCT, antiviral therapy, and targeted antibody therapy. In particular, mogamulizumab is emerging as a promising new therapy, either alone or in combination with other therapies. Treatment with mogamulizumab and standard chemotherapy to induce higher rates of CR may benefit the long-term survival of patients with ATLL who are eligible for allo-HSCT. In addition, mogamulizumab may be used to rescue patients who have relapsed after transplantation. ${ }^{32} \mathrm{As}$ mogamulizumab depletes Treg cells, the positive and negative immunological effects of the use of mogamulizumab need to be carefully examined, especially for patients who undergo allo-HSCT. Mogamulizumab has a less toxic profile than conventional cytotoxic chemotherapies and induces a high response rate in patients with ATLL, even in elderly patients. Therefore, it may provide an effective treatment option for the disease, especially for elderly patients who are not eligible for intensive chemotherapy or hematopoietic stem-cell transplantation. Determining the optimal combination of mogamulizumab with conventional and novel therapies is expected to improve the prognosis of patients with ATLL.

\section{Disclosure}

The authors report no conflicts of interest in this work.

\section{References}

1. Uchiyama T, Yodoi J, Sagawa K, Takatsuki K, Uchino H. Adult T-cell leukemia: clinical and hematologic features of 16 cases. Blood. 1977;50(3):481-492.
2. Hinuma Y, Nagata K, Hanaoka M, et al. Adult T-cell leukemia: antigen in an ATL cell line and detection of antibodies to the antigen in human sera. Proc Natl Acad Sci U S A. 1981;78(10):6476-6480.

3. Yoshida M, Miyoshi I, Hinuma Y. Isolation and characterization of retrovirus from cell lines of human adult T-cell leukemia and its implication in the disease. Proc Natl Acad Sci U S A. 1982;79(6): 2031-2035.

4. Poiesz BJ, Ruscetti FW, Gazdar AF, Bunn PA, Minna JD, Gallo RC. Detection and isolation of type $\mathrm{C}$ retrovirus particles from fresh and cultured lymphocytes of a patient with cutaneous T-cell lymphoma. Proc Natl Acad Sci U S A. 1980;77(12):7415-7419.

5. Katsuya H, Yamanaka T, Ishitsuka K, et al. Prognostic index for acuteand lymphoma-type adult T-cell leukemia/lymphoma. J Clin Oncol. 2012;30(14):1635-1640.

6. Shigematsu A, Kobayashi N, Yasui H, et al. High level of serum soluble interleukin-2 receptor at transplantation predicts poor outcome of allogeneic stem cell transplantation for adult T cell leukemia. Biol Blood Marrow Transplant. 2014;20(6):801-805.

7. Satake M, Yamada Y, Atogami S, Yamaguchi K. The incidence of adult T-cell leukemia/lymphoma among HTLV-1 carriers in Japan. Leuk Lymphoma. 2014;1-28.

8. Tsukasaki K, Hermine O, Bazarbachi A, et al. Definition, prognostic factors, treatment, and response criteria of adult T-cell leukemialymphoma: a proposal from an international consensus meeting. J Clin Oncol. 2009;27(3):453-459.

9. Tsukasaki K, Utsunomiya A, Fukuda H, et al; Japan Clinical Oncology Group Study JCOG9801. VCAP-AMP-VECP compared with biweekly CHOP for adult T-cell leukemia-lymphoma: Japan Clinical Oncology Group Study JCOG9801. J Clin Oncol. 2007;25(34):5458-5464.

10. Utsunomiya A, Miyazaki Y, Takatsuka Y, et al. Improved outcome of adult $\mathrm{T}$ cell leukemia/lymphoma with allogeneic hematopoietic stem cell transplantation. Bone Marrow Transplant. 2001;27(1):15-20.

11. Choi I, Tanosaki R, Uike N, et al; ATLL allo-HSCT Study Group. Long-term outcomes after hematopoietic SCT for adult T-cell leukemia/ lymphoma: results of prospective trials. Bone Marrow Transplant. 2011;46(1):116-118.

12. Hishizawa M, Kanda J, Utsunomiya A, et al. Transplantation of allogeneic hematopoietic stem cells for adult T-cell leukemia: a nationwide retrospective study. Blood. 2010;116(8):1369-1376.

13. Kanda J, Hishizawa M, Utsunomiya A, et al. Impact of graft-versus-host disease on outcomes after allogeneic hematopoietic cell transplantation for adult T-cell leukemia: a retrospective cohort study. Blood. 2012;119(9):2141-2148.

14. Ishida $\mathrm{T}$, Hishizawa $\mathrm{M}$, Kato $\mathrm{K}$, et al. Allogeneic hematopoietic stem cell transplantation for adult T-cell leukemia-lymphoma with special emphasis on preconditioning regimen: a nationwide retrospective study. Blood. 2012;120(8):1734-1741.

15. Bazarbachi A, Plumelle Y, Carlos Ramos J, et al. Meta-analysis on the use of zidovudine and interferon-alfa in adult T-cell leukemia/ lymphoma showing improved survival in the leukemic subtypes. J Clin Oncol. 2010;28(27):4177-4183.

16. Coiffier B, Lepage E, Briere J, et al. CHOP chemotherapy plus rituximab compared with $\mathrm{CHOP}$ alone in elderly patients with diffuse large-B-cell lymphoma. N Engl J Med. 2002;346(4):235-242.

17. Yoshie O, Matsushima K. CCR4 and its ligands: from bench to bedside. Int Immunol. Epub 2014 Aug 2.

18. Yoshie O, Fujisawa R, Nakayama T, et al. Frequent expression of CCR4 in adult T-cell leukemia and human T-cell leukemia virus type 1-transformed T cells. Blood. 2002;99(5):1505-1511.

19. Ishida $\mathrm{T}$, Utsunomiya $\mathrm{A}$, Iida $\mathrm{S}$, et al. Clinical significance of CCR4 expression in adult T-cell leukemia/lymphoma: its close association with skin involvement and unfavorable outcome. Clin Cancer Res. 2003;9(10 Pt 1):3625-3634.

20. Clynes RA, Towers TL, Presta LG, Ravetch JV. Inhibitory Fc receptors modulate in vivo cytotoxicity against tumor targets. Nat Med. 2000;6(4): 443-446. 
21. Shields RL, Lai J, Keck R, et al. Lack of fucose on human IgG1 N-linked oligosaccharide improves binding to human Fcgamma RIII and antibody-dependent cellular toxicity. J Biol Chem. 2002;277(30): 26733-26740.

22. Niwa R, Sakurada M, Kobayashi Y, et al. Enhanced natural killer cell binding and activation by low-fucose $\mathrm{IgG} 1$ antibody results in potent antibody-dependent cellular cytotoxicity induction at lower antigen density. Clin Cancer Res. 2005;11(6):2327-2336.

23. Niwa R, Shoji-Hosaka E, Sakurada M, et al. Defucosylated chimeric anti-CC chemokine receptor 4 IgG1 with enhanced antibody-dependent cellular cytotoxicity shows potent therapeutic activity to T-cell leukemia and lymphoma. Cancer Res. 2004;64(6):2127-2133.

24. Ishida T, Iida S, Akatsuka Y, et al. The CC chemokine receptor 4 as a novel specific molecular target for immunotherapy in adult T-Cell leukemia/lymphoma. Clin Cancer Res. 2004;10(22):7529-7539.

25. Ishii $T$, Ishida $T$, Utsunomiya A, et al. Defucosylated humanized anti-CCR4 monoclonal antibody KW-0761 as a novel immunotherapeutic agent for adult T-cell leukemia/lymphoma. Clin Cancer Res. 2010;16(5):1520-1531.

26. Ito A, Ishida T, Yano H, et al. Defucosylated anti-CCR4 monoclonal antibody exercises potent ADCC-mediated antitumor effect in the novel tumor-bearing humanized NOD/Shi-scid, IL-2Rgamma(null) mouse model. Cancer Immunol Immunother. 2009;58(8):1195-1206.

27. Ito A, Ishida T, Utsunomiya A, et al. Defucosylated anti-CCR4 monoclonal antibody exerts potent ADCC against primary ATLL cells mediated by autologous human immune cells in NOD/Shi-scid, IL-2R gamma(null) mice in vivo. J Immunol. 2009;183(7):4782-4791.

28. Yamamoto K, Utsunomiya A, Tobinai K, et al. Phase I study of KW-0761, a defucosylated humanized anti-CCR4 antibody, in relapsed patients with adult T-cell leukemia-lymphoma and peripheral T-cell lymphoma. J Clin Oncol. 2010;28(9):1591-1598.

29. Ishida T, Joh T, Uike N, et al. Defucosylated anti-CCR4 monoclonal antibody (KW-0761) for relapsed adult T-cell leukemia-lymphoma: a multicenter phase II study. J Clin Oncol. 2012;30(8):837-842.

30. Ohgiya D, Machida S, Ichiki A, et al. Strong effect of mogamulizumab on splenic residual disease of adult $\mathrm{T}$ cell leukemia/lymphoma. Ann Hematol. 2014;93(4):719-720.

31. Motohashi K, Suzuki T, Kishimoto K, et al. Successful treatment of a patient with adult $\mathrm{T}$ cell leukemia/lymphoma using anti-CC chemokine receptor 4 monoclonal antibody mogamulizumab followed by allogeneic hematopoietic stem cell transplantation. Int J Hematol. 2013;98(2): 258-260.
32. Ito Y, Miyamoto T, Chong Y, et al. Successful treatment with anti-CC chemokine receptor $4 \mathrm{MoAb}$ of relapsed adult T-cell leukemia/ lymphoma after umbilical cord blood transplantation. Bone Marrow Transplant. 2013;48(7):998-999.

33. Jo T, Ishida T, Takemoto S, et al. Randomized phase II study of mogamulizumab (KW-0761) plus VCAP-AMP-VECP (mLSG15) versus mLSG15 alone for newly diagnosed aggressive adult T-cell leukemialymphoma (ATL). J Clin Oncol. 2013;suppl:abstr 8506.

34. Kanazawa T, Hiramatsu Y, Iwata S, et al. Anti-CCR4 monoclonal antibody mogamulizumab for the treatment of EBV-associated T- and NK-cell lymphoproliferative diseases. Clin Cancer Res. 2014;20(19): 5075-5084.

35. Ogura M, Ishida T, Hatake K, et al. Multicenter phase II study of mogamulizumab (KW-0761), a defucosylated anti-cc chemokine receptor 4 antibody, in patients with relapsed peripheral T-cell lymphoma and cutaneous T-cell lymphoma. J Clin Oncol. 2014;32(11):1157-1163.

36. Ishida T, Ito A, Sato F, et al. Stevens-Johnson Syndrome associated with mogamulizumab treatment of adult T-cell leukemia/lymphoma. Cancer Sci. 2013;104(5):647-650.

37. Yonekura K, Kanzaki T, Gunshin K, et al. Effect of anti-CCR4 monoclonal antibody (mogamulizumab) on adult T-cell leukemia-lymphoma: cutaneous adverse reactions may predict the prognosis. J Dermatol. 2014;41(3):239-244.

38. Nakano N, Kusumoto S, Tanaka Y, et al. Reactivation of hepatitis $\mathrm{B}$ virus in a patient with adult T-cell leukemia-lymphoma receiving the anti-CC chemokine receptor 4 antibody mogamulizumab. Hepatol Res. 2014;44(3):354-357.

39. Ohyama Y, Kumode T, Eguchi G, Yamaguchi T, Maeda Y. Induction of molecular remission by using anti-CC-chemokine receptor 4 (anti-CCR4) antibodies for adult T cell leukemia: a risk of opportunistic infection after treatment with anti-CCR4 antibodies. Ann Hematol. 2014;93(1):169-171.

40. Kato K, Miyamoto T, Numata A, et al. Diffuse panbronchiolitis after humanized anti-CCR4 monoclonal antibody therapy for relapsed adult T-cell leukemia/lymphoma. Int J Hematol. 2013;97(3):430-432.
Blood and Lymphatic Cancer: Targets and Therapy

\section{Publish your work in this journal}

Blood and Lymphatic Cancer: Targets and Therapy is an international, peer-reviewed, open access journal focusing on blood and lymphatic cancer research, identification of therapeutic targets and the optimal use of preventative and integrated treatment interventions to achieve improved outcomes, enhanced survival and quality of life for the

\section{Dovepress}

cancer patient. The manuscript management system is completely online and includes a very quick and fair peer-review system. Visit http://www.dovepress.com/testimonials.php to read real quotes from published authors. 